June 1996

PAR-LPTHE 96/21

\title{
LEPTONIC CUSTODIAL SYMMETRY, QUANTIZATION OF THE ELECTRIC CHARGE AND THE NEUTRINO IN THE STANDARD MODEL.
}

\author{
B. Machet 円曰 \\ Laboratoire de Physique Théorique et Hautes Energies, 3 \\ Universités Pierre et Marie Curie (Paris 6) et Denis Diderot (Paris 7); \\ Unité associée au CNRS URA 280.
}

\begin{abstract}
I study, in the leptonic sector, the role, of the $S U(2)_{V}$ custodial symmetry $\tilde{\mathcal{G}}$ which was shown in ref. [何 to control the quantization of the electric charge in the $J=0$ mesonic sector. The electroweak theory is considered, according to ref. [2], as a purely vectorial model which interacts with a "hidden" sector of composite scalars. $\tilde{\mathcal{G}}$ can only be a symmetry of the former if the neutrino is a Majorana particle; the latter provides a dynamical modification of the leptonic weak couplings, reconstructing those of the Standard Model with a massless Majorana neutrino.
\end{abstract}

PACS: 11.15.-q, 11.30.Rd, 13.10.+q, 14.60.Pq

\footnotetext{
${ }^{1}$ Member of 'Centre National de la Recherche Scientifique'.

${ }^{2}$ E-mail: machet@lpthe.jussieu.fr.

${ }^{3}$ LPTHE tour $16 / 1^{\text {er }}$ étage, Université P. et M. Curie, BP 126, 4 place Jussieu, F 75252 PARIS CEDEX 05 (France).
} 


\section{Introduction.}

The eigenvalues of the electric charge operator are naturally quantized if it is one of the generators of a simple group of symmetry of the theory under consideration; it can indeed always be then considered as the " $z$ " component of an $S U(2)$ angular momentum subgroup.

In [1], I constructed a renormalizable, anomaly-free, gauge $S U(2)_{L} \times U(1)$ electroweak theory for $J=0$ mesons by extending the scalar sector of the Standard Model [3], and showed that it has, classically, independently of the hypercharge coupling constant $g^{\prime}$, a global "custodial" $S U(2)_{V}$ symmetry which includes the $U(1)$ group of electromagnetism; it is the (unbroken) diagonal subgroup of the chiral $S U(2)_{L} \times S U(2)_{R}$.

The underlying suggestion there is that the quantization of the electric charge in this sector is the reflection that this custodial $S U(2)_{V}$ is an exact symmetry of the model. The fitting of each complex doublet of mesons into one $S U(2)_{V}$ real triplet with electric charges $(-1,0,+1)$ plus one real chargeless singlet eases the connection between this group and its electromagnetic subgroup.

The case of leptons appears less intuitive since each family, in the Glashow-Salam-Weinberg model [3] is cast into one doublet plus one singlet of the underlying $S U(2)_{L}$ gauge group of symmetry, both including a charged particle; there is clearly a mismatch here between their integer electric charges and half-integer values of $S U(2)_{L}$ " $z$ " quantum numbers.

We nevertheless adopt the unifying point of view that the leptonic quantization of electric charge is the sign that a custodial $S U(2)$ symmetry also operates for asymptotic states.

This sector is studied here in relation with ref. [2]. It is described by a purely vectorial theory which rebuilds the Standard Model by interacting with a "hidden" sector. We are concerned whether the fundamental vectorial theory for the leptonic asymptotic states can satisfy charge quantization, that is whether it can be globally invariant by the same custodial $S U(2)$ as that of the mesonic sector. We do not require this invariance for the whole electroweak theory since the "hidden" sector does not correspond to asymptotic states.

\section{Groups and representations.}

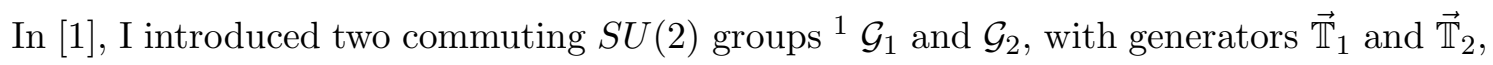
which acted on special (quadruplet) representations of $J=0$ mesons

$$
\left(\mathbb{M}^{0}, \overrightarrow{\mathbb{M}}\right)
$$

as follows (up to an irrelevant global sign):

- on representations "even" by the parity changing operator $\mathcal{P}$ (scalar + pseudoscalar):

$$
\begin{aligned}
\mathbb{T}_{1}^{i} \cdot \mathbb{M}^{j} & =\frac{i}{2}\left(\epsilon_{i j k} \mathbb{M}^{k}+\delta_{i j} \mathbb{M}^{0}\right) \\
\mathbb{T}_{1}^{i} \cdot \mathbb{M}^{0} & =-\frac{i}{2} \mathbb{M}^{i}
\end{aligned}
$$

\footnotetext{
${ }^{1}$ called there "left" and "right", but we denote them differently here to avoid confusion.
} 
and

$$
\begin{aligned}
\mathbb{T}_{2}^{i} \cdot \mathbb{M}^{j} & =\frac{i}{2}\left(\epsilon_{i j k} \mathbb{M}^{k}-\delta_{i j} \mathbb{M}^{0}\right) \\
\mathbb{T}_{2}^{i} \cdot \mathbb{M}^{0} & =\frac{i}{2} \mathbb{M}^{i}
\end{aligned}
$$

- on representations "odd" (scalar - pseudoscalar), the roles of the two groups are swapped: $\mathcal{G}_{1}$ acts on "odd" representations like $\mathcal{G}_{2}$ on "even" representations, and viceversa.

The dot "." between a generator and a field $\mathbb{M}$ stands for the action of the former on the latter. The Latin indices $i, j, k$ run from 1 to 3 , and we also define $\mathbb{M}^{ \pm}=\left(\mathbb{M}^{1} \pm i \mathbb{M}^{2}\right) / \sqrt{2}$, $\mathbb{T}^{ \pm}=\mathbb{T}^{1} \pm i \mathbb{T}^{2}$.

The quadruplets are reducible representations of each of these two groups, and can be decomposed into two spin $1 / 2$ doublets. With respect to the diagonal $S U(2)_{V}$ subgroup $\tilde{\mathcal{G}}$ of the chiral group $\mathcal{G}_{1} \times \mathcal{G}_{2}$, they decompose differently, into one spin 1 triplet, $\overrightarrow{\mathbb{M}}$, plus one singlet $\mathbb{M}^{0}$. $\tilde{\mathcal{G}}$ is the custodial symmetry which occurs in the mesonic sector. The generator of the $U(1)$ group of electromagnetism is the " $z$ " generator of this angular momentum.

Let us now consider, for each generation of leptons, the left-handed \& quadruplet of neutrinos and charged leptons (we do not question $e-\mu-\tau$ universality and all leptons are a priori 4-components (chiral) leptons)

$$
\mathcal{Q}_{L}=\left(\mathbb{L}^{0}, \mathbb{L}^{3}, \mathbb{L}^{+}, \mathbb{L}^{-}\right)=\left(-i \frac{\nu-\nu^{c}}{\sqrt{2}}, \frac{\nu+\nu^{c}}{\sqrt{2}}, \ell^{+}, \ell^{-}\right)_{L}
$$

$\ell^{+}$and $\ell^{-}, \nu^{c}$ and $\nu$ are charge conjugate:

$$
\ell^{+}=C{\overline{\ell^{-}}}^{T}, \quad \nu^{c}=C \bar{\nu}^{T}
$$

the superscript " $T$ " means "transposed" and $C$ is the charge-conjugation operator: $C=$ $i \gamma_{2} \gamma_{0}$ in the Dirac representation. The convention that $\ell^{+}=\left(\ell^{1}+i \ell^{2}\right) / \sqrt{2}$ is the charge conjugate of $\ell^{-}=\left(\ell^{1}-i \ell^{2}\right) / \sqrt{2}$ entails that $i$ gives $-i$ by charge conjugation and that the charge conjugate of $\mathcal{Q}_{L}$ is its right-handed counterpart $\mathcal{Q}_{R}=\left(\mathbb{R}^{0}, \overrightarrow{\mathbb{R}}\right)$.

We define the actions of $\mathcal{G}_{1}$ and $\mathcal{G}_{2}$ on $\mathcal{Q}_{\mathcal{L}}$ by eqs. (2) and (3).

$\mathcal{Q}_{L}$ can be decomposed into:

- two doublets of $\mathcal{G}_{1}$ :

$$
l_{1}=\left(\begin{array}{c}
\frac{1}{\sqrt{2}}\left(\mathbb{L}^{3}+i \mathbb{L}^{0}\right) \\
\mathbb{L}^{-}
\end{array}\right)=\left(\begin{array}{c}
\nu \\
\ell^{-}
\end{array}\right)_{L}, \quad l_{1}^{\prime}=\left(\begin{array}{c}
\mathbb{L}^{+} \\
\frac{1}{\sqrt{2}}\left(\mathbb{L}^{3}-i \mathbb{L}^{0}\right)
\end{array}\right)=\left(\begin{array}{c}
\ell^{+} \\
\nu^{c}
\end{array}\right)_{L}
$$

with the group action

$$
\begin{aligned}
& \mathbb{T}_{1}^{3} \cdot \ell_{L}^{-}=-\frac{1}{2} \ell_{L}^{-}, \quad \mathbb{T}_{1}^{3} \cdot \ell_{L}^{+}=\frac{1}{2} \ell_{L}^{+}, \quad \mathbb{T}_{1}^{3} \cdot \nu_{L}=\frac{1}{2} \nu_{L}, \quad \mathbb{T}_{1}^{3} \cdot\left(\nu^{c}\right)_{L}=-\frac{1}{2}\left(\nu^{c}\right)_{L}, \\
& \mathbb{T}_{1}^{+} \cdot \ell_{L}^{-}=\nu_{L}, \quad \mathbb{T}_{1}^{+} \cdot \ell_{L}^{+}=0, \quad \mathbb{T}_{1}^{+} \cdot \nu_{L}=0, \quad \mathbb{T}_{1}^{+} \cdot\left(\nu^{c}\right)_{L}=-\ell_{L}^{+}, \\
& \mathbb{T}_{1}^{-} \cdot \ell_{L}^{-}=0, \quad \mathbb{T}_{1}^{-} \cdot \ell_{L}^{+}=-\left(\nu^{c}\right)_{L}, \quad \mathbb{T}_{1}^{-} \cdot \nu_{L}=\ell_{L}^{\frac{1}{L}}, \quad \mathbb{T}_{1}^{-} \cdot\left(\nu^{c}\right)_{L}=0
\end{aligned}
$$

\footnotetext{
${ }^{2}$ We shall use hereafter the subscript " $L$ " for "left-handed" fermions, and " $R$ " for "right-handed" ones.
} 
$\mathcal{G}_{1}$ acts on $l_{1}$ like the $S U(2)_{L}$ group of the Standard Model.

- two doublets of $\mathcal{G}_{2}$ :

$$
l_{2}=\left(\begin{array}{c}
\nu^{c} \\
\ell^{-}
\end{array}\right)_{L}, \quad l_{2}^{\prime}=\left(\begin{array}{c}
\ell^{+} \\
\nu
\end{array}\right)_{L}
$$

with the group action

$$
\begin{aligned}
& \mathbb{T}_{2}^{3} \cdot \ell_{L}^{-}=-\frac{1}{2} \ell_{L}^{-}, \quad \mathbb{T}_{2}^{3} \cdot \ell_{L}^{+}=\frac{1}{2} \ell_{L}^{+}, \quad \mathbb{T}_{2}^{3} \cdot \nu_{L}=-\frac{1}{2} \nu_{L}, \quad \mathbb{T}_{2}^{3} \cdot\left(\nu^{c}\right)_{L}=\frac{1}{2}\left(\nu^{c}\right)_{L}, \\
& \mathbb{T}_{2}^{+} \cdot \ell_{L}^{-}=\left(\nu^{c}\right)_{L}, \quad \mathbb{T}_{2}^{+} \cdot \ell_{L}^{+}=0, \quad \mathbb{T}_{2}^{+} \cdot \nu_{L}=-\ell_{L}^{+}, \quad \mathbb{T}_{2}^{+} \cdot\left(\nu^{c}\right)_{L}=0, \\
& \mathbb{T}_{2}^{-} \cdot \ell_{L}^{-}=0, \quad \mathbb{T}_{2}^{-} \cdot \ell_{L}^{+}=-\nu_{L}, \quad \mathbb{T}_{2}^{-} \cdot \nu_{L}=0, \quad \mathbb{T}_{2}^{-} \cdot\left(\nu^{c}\right)_{L}=\ell_{L}^{-}
\end{aligned}
$$

- one triplet $\left(\mathbb{L}^{3}, \mathbb{L}^{+}, \mathbb{L}^{-}\right)$plus one singlet $\mathbb{L}^{0}$ of the diagonal $S U(2)_{V}$ with generators $\tilde{\mathbb{T}}^{i}=\mathbb{T}_{1}^{i}+\mathbb{T}_{2}^{i}$, with the group action

$$
\begin{aligned}
& \tilde{\mathbb{T}}^{3} \cdot \ell_{L}^{-}=-\ell_{L}^{-}, \quad \tilde{\mathbb{T}}^{3} \cdot \ell_{L}^{+}=\ell_{L}^{+}, \quad \tilde{\mathbb{T}}^{3} \cdot \nu_{L}=0, \quad \tilde{\mathbb{T}}^{3} \cdot\left(\nu^{c}\right)_{L}=0, \\
& \tilde{\mathbb{T}}^{+} \cdot \ell_{L}^{-}=\nu_{L}+\left(\nu^{c}\right)_{L}, \quad \tilde{\mathbb{T}}^{+} \cdot \ell_{L}^{+}=0, \quad \tilde{\mathbb{T}}^{+} \cdot \nu_{L}=-\ell_{L}^{+}, \quad \tilde{\mathbb{T}}^{+} \cdot\left(\nu^{c}\right)_{L}=-\ell_{L}^{+}, \\
& \tilde{\mathbb{T}}^{-} \cdot \ell_{L}^{-}=0, \quad \tilde{\mathbb{T}}^{-} \cdot \ell_{L}^{+}=-\left(\nu_{L}+\left(\nu^{c}\right)_{L}\right), \quad \tilde{\mathbb{T}}^{-} \cdot \nu_{L}=\ell_{L}^{-}, \quad \tilde{\mathbb{T}}^{-} \cdot\left(\nu^{c}\right)_{L}=\ell_{L}^{-} .
\end{aligned}
$$

When operating in the 4-dimensional vector space spanned by the four entries of $\mathcal{Q}_{L}$, its three generators write as $4 \times 4$ matrices (see eq. (28) of ref. [1]), according to (in the basis $\left.\left(\mathbb{L}^{0}, \mathbb{L}^{3}, \mathbb{L}^{+} \mathbb{L}^{-}\right)\right)$:

$$
\tilde{\mathbb{T}}^{+}=\left(\begin{array}{cccc}
0 & 0 & 0 & 0 \\
0 & 0 & -\sqrt{2} & 0 \\
0 & 0 & 0 & 0 \\
0 & \sqrt{2} & 0 & 0
\end{array}\right), \quad \tilde{\mathbb{T}}^{-}=\left(\begin{array}{cccc}
0 & 0 & 0 & 0 \\
0 & 0 & 0 & \sqrt{2} \\
0 & -\sqrt{2} & 0 & 0 \\
0 & 0 & 0 & 0
\end{array}\right), \quad \tilde{\mathbb{T}}^{3}=\left(\begin{array}{cccc}
0 & 0 & 0 & 0 \\
0 & 0 & 0 & 0 \\
0 & 0 & 1 & 0 \\
0 & 0 & 0 & -1
\end{array}\right) ;
$$

The electric charge generator is identical with the third generator of $S U(2)_{V}$ :

$$
\mathbb{Q}=\tilde{\mathbb{T}}^{3}
$$

By operating with charge conjugation on the transformed, by an element of the group, of an entry of $\mathcal{Q}_{L}$, one deduces that its charge conjugate $\mathcal{Q}_{R}$ also transforms according to eqs. (2, 3). The decompositions above thus apply to $\mathcal{Q}_{R}$ too.

To each quadruplet of leptons is associated a quadratic scalar expression invariant by all $S U(2)$ groups considered above

$$
\mathcal{I}=\overline{\mathcal{Q}} \mathcal{Q}=\overline{\mathcal{Q}_{R}} \mathcal{Q}_{L}+\overline{\mathcal{Q}_{L}} \mathcal{Q}_{R}
$$

\section{Chiral symmetry and vector-like Lagrangians for leptons.}

We intend to make the link between a vectorial Lagrangian like that in [2] and a chiral symmetry $\mathcal{G}_{1} \times \mathcal{G}_{2}$ like that occurring naturally in [1]. I show here that such a vectorial Lagrangian can be considered as a $\mathcal{G}_{1} \times U(1)\left(\right.$ or $\left.\mathcal{G}_{2} \times U(1)\right)$ gauge theory for $\mathcal{Q}_{L}$ or $\mathcal{Q}_{R}$. 
The chiral symmetry then naturally springs out at the limit when the two electroweak couplings vanish.

Let us construct a $\mathcal{G}_{1} \times U(1)$ gauge theory for $\mathcal{Q}_{L}$. The three $\mathcal{G}_{1}$ gauge fields form the triplet $\vec{W}_{\mu}$, and the $U(1)$ gauge field is $B_{\mu}$.

The generator $\mathbb{Y}$ of the $U(1)$ group is taken to satisfy the Gell-Mann-Nishijima relation

$$
\mathbb{Y}=\mathbb{Q}-\mathbb{T}_{1}^{3}
$$

The $U(1)$ is a gauging of the leptonic number; indeed, the leptonic numbers of the entries of $\mathcal{Q}$ are $(-2) \times$ their $U(1)$ quantum numbers.

The notations are, as usual

$$
Z_{\mu}=c_{w} W_{\mu}^{3}-s_{w} B_{\mu}, A_{\mu}=c_{w} B_{\mu}+s_{w} W_{\mu}^{3},
$$

where $c_{w}$ and $s_{w}$ are the cosine and sine of the Weinberg angle; $A_{\mu}$ is the photon; $W_{\mu}^{ \pm}=$ $\left(W_{\mu}^{1} \pm W_{\mu}^{2}\right) / \sqrt{2} ; g=e / s_{w}$ and $g^{\prime}=e / c_{w}$ are respectively the $\mathcal{G}_{1}$ and $U(1)$ coupling constants.

The Lagrangian is the sum of two Lagrangians $\mathcal{L}_{1}$ and $\mathcal{L}_{1}^{\prime}$ corresponding respectively to the two doublets $l_{1}$ and $l_{1}^{\prime}$. We use the abbreviated notation $\gamma_{\mu L}=\gamma_{\mu}\left(1-\gamma_{5}\right) / 2, \gamma_{\mu R}=$ $\gamma_{\mu}\left(1+\gamma_{5}\right) / 2$.

$$
\begin{aligned}
\mathcal{L}_{1}= & \overline{i \ell^{-}} \gamma_{L}^{\mu} \partial_{\mu} \ell^{-}+i \bar{\nu} \gamma_{L}^{\mu} \partial_{\mu} \nu \\
& +\frac{e}{\sqrt{2} s_{w}}\left(\overline{\ell^{-}} \gamma_{L}^{\mu} W_{\mu}^{-} \nu+\bar{\nu} \gamma_{L}^{\mu} W_{\mu}^{+} \ell^{-}\right) \\
& -\frac{e}{2 s_{w}}\left(\overline{\ell^{-}} \gamma_{L}^{\mu} W_{\mu}^{3} \ell^{-}-\bar{\nu} \gamma_{L}^{\mu} W_{\mu}^{3} \nu\right) \\
& -\frac{e}{2 c_{w}}\left(\overline{\ell^{-}} \gamma_{L}^{\mu} B_{\mu} \ell^{-}+\bar{\nu} \gamma_{L}^{\mu} B_{\mu} \nu\right) ; \\
\mathcal{L}_{1}^{\prime}=\quad & i \overline{\ell^{+}} \gamma_{L}^{\mu} \partial_{\mu} \ell^{+}+i \overline{\nu^{c}} \gamma_{L}^{\mu} \partial_{\mu} \nu^{c} \\
& -\frac{e}{\sqrt{2} s_{w}}\left(\overline{\ell^{+}} \gamma_{L}^{\mu} W_{\mu}^{+} \nu^{c}-\overline{\nu^{c}} \gamma_{L}^{\mu} W_{\mu}^{-} \ell^{+}\right) \\
+ & \frac{e}{2 s_{w}}\left(\overline{\ell^{+}} \gamma_{L}^{\mu} W_{\mu}^{3} \ell^{+}-\overline{\nu^{c}} \gamma_{L}^{\mu} W_{\mu}^{3} \nu^{c}\right) \\
+ & \frac{e}{2 c_{w}}\left(\overline{\ell^{+}} \gamma_{L}^{\mu} B_{\mu} \ell^{+}+\overline{\nu^{c}} \gamma_{L}^{\mu} B_{\mu} \nu^{c}\right) .
\end{aligned}
$$

Using the properties of charge conjugation in $\mathcal{L}_{1}^{\prime}, \mathcal{L}_{1}+\mathcal{L}_{1}^{\prime}$ can be cast into the purely vectorial Lagrangian of [2]; it is a $S U(2) \times U(1)$ gauge Lagrangian for a $\left(\nu, \ell^{-}\right)$doublet:

$$
\begin{aligned}
\mathcal{L}=\mathcal{L}_{1}+\mathcal{L}_{1}^{\prime}= & \overline{i \ell^{-}} \gamma^{\mu} \partial_{\mu} \ell^{-}+i \bar{\nu} \gamma^{\mu} \partial_{\mu} \nu \\
& +\frac{e}{\sqrt{2} s_{w}}\left(\overline{\ell^{-}} \gamma^{\mu} W_{\mu}^{-} \nu+\bar{\nu} \gamma^{\mu} W_{\mu}^{+} \ell^{-}\right) \\
& -\frac{e}{2 s_{w}}\left(\overline{\ell^{-}} \gamma^{\mu} W_{\mu}^{3} \ell^{-}-\bar{\nu} \gamma^{\mu} W_{\mu}^{3} \nu\right) \\
& -\frac{e}{2 c_{w}}\left(\overline{\ell^{-}} \gamma^{\mu} B_{\mu} \ell^{-}+\bar{\nu} \gamma^{\mu} B_{\mu} \nu\right) .
\end{aligned}
$$

The same result can be obtained by considering $\mathcal{Q}_{R}$ instead of $\mathcal{Q}_{L}$. 
To $\mathcal{L}$ we can add the mass term

$$
\mathcal{L}_{m}=-\frac{m}{2}\left(\overline{\mathcal{Q}_{R}} \mathcal{Q}_{L}+\overline{\mathcal{Q}_{L}} \mathcal{Q}_{R}\right) .
$$

The quadratic expression (13) being invariant by both $\mathcal{G}_{1}$ and $\mathcal{G}_{2}, \mathcal{L}_{m}$ is invariant by the chiral group $\mathcal{G}_{1} \times \mathcal{G}_{2}$, and this invariance is independent of the mass $m$, which can vary with the leptonic generation.

$\mathcal{L}_{m}$ corresponds to a Dirac mass term. It is an important actor in the "see-saw" mechanism evoked in the last section. A Majorana mass term for the neutrino would correspond to the combination (forgetting the charged leptons) $\left(\overline{\mathbb{R}^{3}} \mathbb{L}^{3}+\overline{\mathbb{L}^{3}} \mathbb{R}^{3}\right)-\left(\overline{\mathbb{R}^{0}} \mathbb{L}^{0}+\overline{\mathbb{L}^{0}} \mathbb{R}^{0}\right)+\cdots$, (the "-" sign makes the difference), which is not invariant by $\mathcal{G}_{1}$.

Would we make a similar construction with the gauge group $\mathcal{G}_{2} \times U(1)$, we would obtain a Lagrangian similar to (18) but with $\nu$ and $\nu^{c}$ swapped. As this leaves the kinetic terms unaltered, $\mathcal{L}+\mathcal{L}_{m}$ has a global $\mathcal{G}_{1} \times \mathcal{G}_{2}$ chiral symmetry at the limit $g, g^{\prime} \rightarrow 0$, hence also a global symmetry by the diagonal $\tilde{\mathcal{G}}$.

\section{The custodial $S U(2)_{V}$ symmetry.}

Non-vanishing values of $g$ and $g^{\prime}$ break the $\mathcal{G}_{1} \times \mathcal{G}_{2}$ chiral symmetry. We ask at which condition the diagonal group $\tilde{\mathcal{G}}$ can stay an unbroken global symmetry of $\mathcal{L}+\mathcal{L}_{m}$, providing an understanding of the quantization of the electric charge for the electroweak vector-like model under scrutiny, with the same origin as in the mesonic sector [1].

We can rewrite $\mathcal{L}_{1}+\mathcal{L}_{1}^{\prime}$ in the form

$$
\begin{aligned}
& 2\left(\mathcal{L}_{1}+\mathcal{L}_{1}^{\prime}\right)= \\
& i\left(\overline{\mathbb{L}^{+}} \gamma_{\mu} \partial^{\mu} \mathbb{L}^{+}+\overline{\mathbb{L}^{-}} \gamma_{\mu} \partial^{\mu} \mathbb{L}^{-}+\overline{\mathbb{L}^{3}} \gamma_{\mu} \partial^{\mu} \mathbb{L}^{3}+\overline{\mathbb{L}^{0}} \gamma_{\mu} \partial^{\mu} \mathbb{L}^{0}\right) \\
& +g\left(\overline{\mathbb{L}^{-}} \gamma_{\mu L}\left(\frac{1}{\sqrt{2}}\left(W_{\mu}^{-} \tilde{\mathbb{T}}^{+}+W_{\mu}^{+} \tilde{\mathbb{T}}^{-}\right)+\frac{Z_{\mu}}{c_{w}} \tilde{\mathbb{T}}^{3}\right) \cdot \mathbb{L}^{-}\right. \\
& +\overline{\mathbb{L}^{+}} \gamma_{\mu L}\left(\frac{1}{\sqrt{2}}\left(W_{\mu}^{-} \tilde{\mathbb{T}}^{+}+W_{\mu}^{+} \tilde{\mathbb{T}}^{-}\right)+\frac{Z_{\mu}}{c_{w}} \tilde{\mathbb{T}}^{3}\right) \cdot \mathbb{L}^{+} \\
& \left.+\overline{\mathbb{L}^{3}} \gamma_{\mu L}\left(\frac{1}{\sqrt{2}}\left(W_{\mu}^{-} \tilde{\mathbb{T}}^{+}+W_{\mu}^{+} \tilde{\mathbb{T}}^{-}\right)+\frac{Z_{\mu}}{c_{w}} \tilde{\mathbb{T}}^{3}\right) \cdot \mathbb{L}^{3}\right) \\
& +i g\left(-\overline{\mathbb{L}^{0}}\left(\gamma_{\mu L} \frac{Z_{\mu}}{c_{w}} \mathbb{L}^{3}+\gamma_{\mu L} W_{\mu}^{-} \mathbb{L}^{+}+\gamma_{\mu L} W_{\mu}^{+} \mathbb{L}^{-}\right)\right. \\
& \left.+\left(\overline{\mathbb{L}^{3}} \gamma_{\mu L} \frac{Z_{\mu}}{c_{w}}+\overline{\mathbb{L}^{-}} \gamma_{\mu L} W_{\mu}^{-}+\overline{\mathbb{L}^{+}} \gamma_{\mu L} W_{\mu}^{+}\right) \mathbb{L}^{0}\right) \\
& +g^{\prime}\left(\overline{\mathbb{L}^{+}} \gamma_{\mu L} B^{\mu} \mathbb{L}^{+}-\overline{\mathbb{L}^{-}} \gamma_{\mu L} B^{\mu} \mathbb{L}^{-}\right),
\end{aligned}
$$

where we have used the fact that $\tilde{\mathbb{T}}^{+}$does not act on $\mathbb{L}^{+}$, nor $\tilde{\mathbb{T}}^{-}$on $\mathbb{L}^{-}$, nor $\tilde{\mathbb{T}}^{3}$ on $\mathbb{L}^{3}$.

The kinetic terms and the second line of (20) are globally $S U(2)_{V}$ invariant when the triplet of gauge bosons $W_{\mu}^{ \pm}$and $Z_{\mu} / c_{w}$ transform, according to [1], like a vector in the adjoint representation of this group.

The next line of $(20)$ is also globally $S U(2)_{V}$ invariant, since $\mathbb{M}^{0}$ and $\overline{\mathbb{M}^{0}}$ are singlets and are each multiplied by another singlet made by the scalar product of two triplets.

Now, $B_{\mu}$ being considered [1] as a singlet of $S U(2)_{V}$, the last line of (20) only becomes $S U(2)_{V}$ invariant if, as can be seen by performing an explicit transformation and using (110.11)

$$
\nu+\nu^{c}=0
$$


i.e. the neutrino has to be a Majorana particle, with only one helicity (or chirality), which can be written [5], in the 4-component notation, either $\gamma_{5} \chi$ or $\gamma_{5} \omega$ with

$$
\chi=\left(\begin{array}{c}
\psi_{L} \\
-\sigma^{2} \psi_{L}^{*}
\end{array}\right), \quad \omega=\left(\begin{array}{c}
\psi_{R} \\
-\sigma^{2} \psi_{R}^{*}
\end{array}\right) .
$$

$\psi_{L}\left(\right.$ resp. $\left.\psi_{R}\right)$ is a two-component Weyl spinor transforming like a $(1 / 2,0)(\operatorname{resp} .(0,1 / 2))$ representation of the Lorentz group; $\sigma^{2}$ is the second Pauli matrix and the superscript “*” means "complex conjugation"; $\sigma^{2} \psi_{L}^{*}\left(\right.$ resp. $\left.\sigma^{2} \psi_{R}^{*}\right)$ transforms like a $(0,1 / 2)$ (resp. $(1 / 2,0))$ representation.

We thus conclude that:

The leptonic Lagrangian $\mathcal{L}$ can have a global custodial $S U(2)_{V}$ symmetry only if the neutrino is a Majorana particle.

Clearly, this condition is not compatible with the decomposition (6) and the corresponding laws of transformation (2). In particular, it requires that the $U(1)$ leptonic number be not conserved. We shall see in the next section how the necessary modifications can occur dynamically with the introduction of a "hidden" sector, along the lines of [2].

Like in [1], we now ask whether the custodial symmetry can become a local symmetry, with the triplet $W_{\mu}^{ \pm}, Z_{\mu} / c_{w}$ transforming like the corresponding gauge potentials. The first two lines of (20) have been intentionally written as those of an $S U(2)_{V}$ gauge theory and are thus naturally locally invariant.

By such a local transformation with parameters $\vec{\zeta}(x)$, the third line varies by

$$
\delta=\vec{J}^{\mu} \mathcal{D}_{\mu} \vec{\zeta}
$$

where $\mathcal{D}_{\mu}$ is the covariant derivative with respect to $S U(2)_{V}$

$$
\mathcal{D}_{\mu} \mathbb{L}=\partial_{\mu} \mathbb{L}-i g\left(\frac{1}{\sqrt{2}}\left(W_{\mu}^{+} \tilde{\mathbb{T}}^{-}+W_{\mu}^{-} \tilde{\mathbb{T}}^{+}\right)+\frac{Z_{\mu}}{c_{w}} \tilde{\mathbb{T}}^{3}\right) \cdot \mathbb{L},
$$

and $\vec{J}_{\mu}$ is a triplet of currents

$$
J_{\mu}^{i}=\overline{\mathbb{L}^{i}} \gamma_{L}^{\mu} \mathbb{L}^{0}-\overline{\mathbb{L}^{0}} \gamma_{L}^{\mu} \mathbb{L}^{i}, \quad i=1,2,3
$$

These currents are covariantly with respect to $\mathcal{G}_{1} \times U(1)$ conserved. As they form a triplet of $S U(2)_{V}$ and because the corresponding $J_{\mu}^{0}$ identically vanishes by definition, one has

$$
D^{\mu} J_{\mu}^{i}=\mathcal{D}^{\mu} J_{\mu}^{i}-i g^{\prime} B_{\mu} \tilde{\mathbb{Q}} \cdot J_{\mu}^{i},
$$

such that the invariance of the third line of (20), requiring the vanishing of $\delta$ up to a total divergence, can only occur when

$$
g^{\prime} B_{\mu} \tilde{\mathbb{Q}} \cdot J_{\mu}^{i}=0, \quad i=1,2,3,
$$

that is when $g^{\prime} \rightarrow 0$.

At this limit, the last line of (20), which vanishes, is also trivially invariant.

We thus find the same result as in [1] for $J=0$ mesons:

The custodial $S U(2)_{L}$ symmetry becomes local in the leptonic sector at the limit $g^{\prime} \rightarrow 0$. 


\section{Left-right spontaneous symmetry breaking; back to the Standard Model for leptons.}

I now summarize and comment the procedure of [2], by which the vector-like model (18) can be reconciled with the Glashow-Salam-Weinberg Lagrangian for leptons.

As Majorana neutrinos are involved, it is natural to introduce the notation

$$
\begin{aligned}
& \chi=\nu_{L}+\left(\nu_{L}\right)^{c}, \\
& \omega=\nu_{R}+\left(\nu_{R}\right)^{c} ;
\end{aligned}
$$

and to rewrite the Lagrangian $(18)+(19)$ as:

$$
\begin{aligned}
\mathcal{L}+\mathcal{L}_{m} & =\overline{i \ell^{-}} \gamma^{\mu} \partial_{\mu} \ell^{-}+\frac{i}{2} \bar{\chi} \gamma^{\mu} \partial_{\mu} \chi+\frac{i}{2} \bar{\omega} \gamma^{\mu} \partial_{\mu} \omega \\
& +\frac{e}{\sqrt{2} s_{w}}\left(\overline{\ell^{-}} \gamma_{L}^{\mu} W_{\mu}^{-} \chi+\bar{\chi} \gamma_{L}^{\mu} W_{\mu}^{+} \ell^{-}\right) \\
& +\frac{e}{\sqrt{2} s_{w}}\left(\overline{\ell^{-}} \gamma_{R}^{\mu} W_{\mu}^{-} \omega+\bar{\omega} \gamma_{R}^{\mu} W_{\mu}^{+} \ell^{-}\right) \\
& +\frac{e}{2 s_{w}}\left(\bar{\chi} \gamma_{L}^{\mu} W_{\mu}^{3} \chi+\bar{\omega} \gamma_{R}^{\mu} W_{\mu}^{3} \omega-\overline{\ell^{-}} \gamma^{\mu} W_{\mu}^{3} \ell^{-}\right) \\
& -\frac{e}{2 c_{w}}\left(\overline{\ell^{-}} \gamma^{\mu} B_{\mu} \ell^{-}+\bar{\chi} \gamma_{L}^{\mu} B_{\mu} \chi+\bar{\omega} \gamma_{R}^{\mu} B_{\mu} \omega\right) \\
& -\frac{m}{2}\left(\bar{\chi} \omega+\bar{\omega} \chi+2 \overline{\ell^{-}} \ell^{-}\right) .
\end{aligned}
$$

Remark: would we have built the model with the group $\mathcal{G}_{2} \times U(1)$, we would have obtained, instead of eq. (29) $\hat{\mathcal{L}}$, deduced from $\mathcal{L}$ by the exchange of $\chi$ and $\omega$, or, equivalently, by that of the "left" and "right" projectors.

We introduce a scalar composite triplet $\Delta$ with leptonic number 2 :

$$
\Delta=\left(\begin{array}{l}
\Delta^{0} \\
\Delta^{-} \\
\Delta^{--}
\end{array}\right)=\frac{\rho}{\nu^{3}}\left(\begin{array}{c}
\overline{\omega_{L}} \omega_{R} \\
\frac{1}{\sqrt{2}}\left(\overline{\ell_{L}^{+}} \omega_{R}+\overline{\omega_{L}} \ell_{R}^{-}\right) \\
\overline{\ell_{L}^{+}} \ell_{R}^{-}
\end{array}\right)=\frac{\rho}{\nu^{3}}\left(\begin{array}{c}
\overline{\nu^{c}} \frac{1+\gamma_{5}}{2} \nu \\
\frac{1}{\sqrt{2}}\left(\overline{\ell^{+}} \frac{1+\gamma_{5}}{2} \nu+\overline{\nu^{c}} \frac{1+\gamma_{5}}{2} \ell^{-}\right) \\
\overline{\ell^{+}} \frac{1+\gamma_{5}}{2} \ell^{-}
\end{array}\right) .
$$

It is a triplet of $\mathcal{G}_{1}$ but not a representation of $\mathcal{G}_{2}$, nor of $\tilde{\mathcal{G}}$.

Its hermitian conjugate is:

$$
\bar{\Delta}=\left(\begin{array}{c}
\overline{\Delta^{0}} \\
\Delta^{+} \\
\Delta^{++}
\end{array}\right)=\frac{\rho}{\nu^{3}}\left(\begin{array}{c}
\overline{\omega_{R}} \omega_{L} \\
\frac{1}{\sqrt{2}}\left(\overline{\ell_{R}^{-}} \omega_{L}+\overline{\omega_{R}} \ell_{L}^{+}\right) \\
\overline{\ell_{R}^{-}} \ell_{L}^{+}
\end{array}\right)=\frac{\rho}{\nu^{3}}\left(\begin{array}{c}
\bar{\nu} \frac{1-\gamma_{5}}{2} \nu^{c} \\
\frac{1}{\sqrt{2}}\left(\overline{\ell^{-}} \frac{1-\gamma_{5}}{2} \nu^{c}+\bar{\nu} \frac{1-\gamma_{5}}{2} \ell^{+}\right) \\
\overline{\ell^{-}} \frac{1-\gamma_{5}}{2} \ell^{+}
\end{array}\right) .
$$

As soon as the mass of $\omega$ is non vanishing, electroweak vacuum fluctuations like described in fig. 1 of [2] can trigger

$$
\left\langle\Delta^{0}\right\rangle=\left\langle\overline{\Delta^{0}}\right\rangle=\rho .
$$

The choice of an $\langle\bar{\omega} \omega\rangle$ condensate, breaking the symmetry between $\chi$ and $\omega$, spontaneously breaks the "left-right" symmetry, or, equivalently, parity.

It could be thought arbitrary since the same type of vacuum fluctuations can also a priori trigger $\langle\bar{\chi} \chi\rangle \neq 0$. However, the diagrams under consideration vanish with the mass of the 
internal fermion. As, by the see-saw mechanism evoked below, an $\langle\bar{\omega} \omega\rangle$ condensate pushes the $\chi$ mass to 0 at the same time that is pushes the $\omega$ mass to $\infty$, the $\langle\bar{\chi} \chi\rangle$ condensate is then automatically suppressed, and vice-versa. This qualitative explanation forbids the coexistence of both condensates.

The proposed mechanism can also be interpreted along the following lines: by expanding $\gamma_{\mu}$ into $\left(\gamma_{\mu L}+\gamma_{\mu R}\right)$, the vectorial Lagrangian (18) can be considered to be that of an $S U(2)_{L} \times S U(2)_{R} \times U(1)$ gauge model for the doublet $\left(\nu, \ell^{-}\right)$; both $S U(2)$ 's act the same way, with the "left" and "right" gauge fields identified. The "Higgs" multiplet $\Delta$ being a triplet of $S U(2)_{R}$ and of $S U(2)_{L}$ with a non-vanishing leptonic number, the condensation of its neutral component spontaneously breaks both $S U(2)$ 's, and $U(1)$.

For quantizing by the Feynman path integral, a Lagrangian of constraint is introduced to take into account the non-independence of the leptonic and $\Delta$ degrees of freedom $(\Lambda$ is an arbitrary mass scale):

$$
\begin{aligned}
\mathcal{L}_{c}= & \lim _{\beta \rightarrow 0}-\frac{\Lambda^{2}}{2 \beta}\left[\left(\Delta^{0}-\frac{\rho}{\nu^{3}} \overline{\omega_{L}} \omega_{R}\right)\left(\overline{\Delta^{0}}-\frac{\rho}{\nu^{3}} \overline{\omega_{R}} \omega_{L}\right)\right. \\
& \left.+\left(\Delta^{-}-\frac{1}{\sqrt{2}} \frac{\rho}{\nu^{3}}\left(\overline{\omega_{L}} \ell_{R}^{-}+\overline{\ell_{L}^{+}} \omega_{R}\right)\right)\left(\Delta^{+}-\frac{1}{\sqrt{2}} \frac{\rho}{\nu^{3}} \overline{\ell_{R}^{-}} \omega_{L}+\overline{\omega_{R}} \ell_{L}^{+}\right)\right) \\
& \left.+\left(\Delta^{--}-\frac{\rho}{\nu^{3}} \overline{\ell_{L}^{+}} \ell_{R}^{-}\right)\left(\Delta^{++}-\frac{\rho}{\nu^{3}} \overline{\ell_{R}^{-}} \ell_{L}^{+}\right)\right] .
\end{aligned}
$$

It has two types of effects, obtained be resumming diagrams at leading order in an expansion in inverse powers of the number of generations and by building a reshuffled perturbation series:

- it gives $\omega$, through an exact "see-saw" mechanism, an infinite (Majorana) mass, leaving $\chi$ as the only asymptotic neutrino state, identified with the observed one; $\chi$ is massless; the left coupling of the charged $W^{\prime}$ 's to $\chi$ and $\ell^{-}$is the only one left over;

- it modifies, through internal $\omega$ loops, the effective couplings of the neutral $W_{\mu}^{3}$ and $B_{\mu}$ gauge bosons, rebuilding the ones of the Standard Model; internal loops do not alter the above mentioned charged $W^{ \pm}$couplings.

Finally, the right-handed $\ell_{R}^{-}$effectively behaves like a $S U(2)_{L}$ singlet with twice the hypercharge of the left-handed one, and the customary structure of weak currents is recovered, in both the charged and neutral sectors. The $\Delta^{0}$ condensate spontaneously breaks the $S U(2)_{R} \times U(1)$ symmetry down to the $U(1)_{Y}$ of weak hypercharge.

None of the components of $\Delta$ appears as an asymptotic state (the constraints give them an infinite mass) and we do not require electric charge quantization for them. It is the same kind of implicit assumption that we made in the mesonic sector where explaining charge quantization for quarks and their underlying gauge theory (Quantum Chromodynamics) was not sought for. So, we allow a Lagrangian which is not globally $\tilde{\mathcal{G}}$ invariant in the hidden sector; this non-invariance is responsible for that of the Lagrangian of the Standard Model.

Only $\mathcal{L}_{c}$, which couples the latter to asymptotic states, has a global $\tilde{\mathcal{G}}$ invariance; it occurs as soon as the laws of transformations of the components of $\Delta$ and those of the corresponding composite fields are the same.

The kinetic terms for the scalars are constructed on the same group of symmetry $\mathcal{G}_{1} \times$ $U(1)$ as $\mathcal{L}$ itself. $S U(2)_{L}$ is also broken by the condensation of $\Delta^{0}$, which thus weakly contributes to the masses of the gauge fields. However, by the decoupling of the weak hidden sector, the Goldstones of this broken symmetry align with the customary hadronic ones. This decoupling also motivates non introducing other triplets of composite states, 
with $\omega$ replaced by $\chi$, since they would not modify the result: as soon as only one type of condensate can occur, the non-condensing additional scalars would simply fade away without any visible effect.

\section{Conclusion.}

The Glashow-Salam-Weinberg Lagrangian has no conspicuous symmetry which could explain charge quantization for leptons. It is a motivation to consider it as an effective theory, with an underlying more fundamental level at which should be sought an explanation for basic observed features of asymptotic states.

It may look going astray to the reader that, in the mesonic sector [1], we left aside the gauge theory of quarks and gluons to directly investigate an electroweak theory of composite asymptotic states, and here, that we added a questionable hidden sector of composite states to a superbly working Standard Model; this maybe paying a heavy price for a modification which brings new unanswered questions.

On the other side, it is hardly possible to solve all problems at the same time, and one must temporarily accept that filling gaps digs holes elsewhere. In front of fundamental questions unsolved, like precisely the origin of the quantization of the electric charge, I disturbed for a while the harmony of the construction to tackle the problem from another point of view. Developments should follow in subsequent works.

Acknowledgments: the author thanks P. Fayet for comments and suggestions. 


\section{References}

[1] B. MACHET: "Chiral Scalar Fields, Custodial Symmetry in Electroweak $S U(2)_{L} \times$ $U(1)$, and the Quantization of the Electric Charge", preprint PAR-LPTHE 96/20, hep-ph/9606239.

[2] M. BELLON and B. MACHET: "The Standard Model of Leptons as a Purely Vectorial Theory", Phys. Lett. B 313 (1993) 341.

[3] S. L. GLASHOW: Nucl. Phys. 22 (1961) 579;

A. SALAM: in "Elementary Particle Theory: Relativistic Groups and Analyticity" (Nobel symposium No 8), edited by N. Svartholm (Almquist and Wiksell, Stockholm 1968);

S. WEINBERG: Phys. Rev. Lett. 19 (1967) 1224.

[4] P. SIKIVIE, L. SUSSKIND, M. VOLOSHIN and V. ZAKHAROV: Nucl. Phys. B 173 (1980) 189.

[5] P. RAMOND: "Field Theory; a Modern Primer", Frontiers in Physics, Lecture Notes Series 51 (Benjamin/Cummings 1981). 